\title{
Analysis of no-tension structures under monotonic loading through an energy-based method
}

\author{
Matteo Bruggi, Alberto Taliercio \\ Department of Civil and Environmental Engineering, Politecnico di Milano, I20133, Milano, Italy
}

\begin{abstract}
An approach is proposed to estimate the collapse load of linear elastic isotropic no-tension 2D solids. The material is replaced by a suitable equivalent orthotropic material with spatially varying local properties. A non-incremental energy-based algorithm is implemented to define the distribution and the orientation of the equivalent material, minimizing the potential energy so as to achieve a compression-only state of stress. The algorithm is embedded within a numerical procedure that evaluates the collapse mechanism of structural elements under monotonic loading. The accuracy of the method is assessed through comparisons with the "exact" results predicted by limit analysis.
\end{abstract}

Keywords: energy-based methods; no-tension materials; masonry-like bodies; collapse mechanisms; structural optimization.

\section{Introduction}

The safety assessment of the architectural heritage is nowadays an issue of paramount importance, both because of the need of avoiding to put human lives at risk, and because of the economic impact of this priceless heritage, for instance, on tourism industry.

Email addresses: matteo.bruggi@polimi.it (Matteo Bruggi), alberto.taliercio@polimi.it (Alberto Taliercio) 
In most countries, the architectural heritage consists of masonry buildings. Masonry, whether of stone or brick, is well known to be a material with low tensile strength. The inability of transferring significant tensile stresses is the reason for the extensive crack patterns that can be observed in many ancient buildings. The presence of cracks is not necessarily symptomatic of a possible collapse, as stresses can spontaneously attain a purely compressive state, which makes cracked regions unnecessary to the stability of the building.

Many formulations have been proposed in the last decades to analyze masonry structures, at different degrees of accuracy. Inelastic constitutive models, taking plastic strains and/or damage effects into account, were presented e.g. in [18, 20, 23], only to quote a few.

Several authors have proposed to analyze masonry structures using no-tension material models: this is why no-tension materials are sometimes referred to as "masonry-like" materials in the literature $[2,11,15]$. Neglecting the low tensile strength of masonry completely is a simplification that is far from being original: in the sixties of last century, Heyman [17] proposed to specialize limit analysis to masonry structures assuming the compressive strength to be unlimited and the tensile strength to vanish. This schematization is spontaneous for stone masonry, consisting of blocks separated by weak joints, but was adopted by other authors also for brick masonry $[2,4,5]$.

Recently, Angelillo et al. [2] proposed an approach to analyze two-dimensional no-tension bodies subjected to given loads based on the unconstrained minimization of the potential energy with respect to the unknown displacement field. The numerical difficulties related to the enforcement of the no-tension constraint [11] are avoided by giving the strain energy density of the material different expressions, according to the sign of the principal stresses.

This idea was later exploited by Bruggi [9] to re-formulate the analysis of no-tension solids as a topology optimization problem. The equilibrium of a two-dimensional no-tension body is found searching for the distribution of an "equivalent" orthotropic material that minimizes the potential energy of the solid. The orthotropic material exhibits negligible stiffness in any direction along 
which a tensile principal stress must be prevented in the isotropic medium. Similarly to [2], this approach obtains the solution under given loads through a one-shot energy-based optimization, provided that the applied loads are compatible with the no-tension constraint.

In this work, an approach is presented to predict the carrying capacity of no-tension 2D structural elements, starting from that proposed in [9]. A numerical scheme is formulated to estimate the collapse load (and the relevant failure mechanism) of no-tension structures with a number of load steps much lower than that required by conventional incremental analyses.

The layout of the paper is as follows. In Sec. 2 the mathematical problem that allows the no-tension isotropic solid to be analyzed as an equivalent orthotropic medium is briefly recalled. The distribution and the orientation of the orthotropic material are determined by minimizing the elastic strain energy of the solid. In Sec. 3, the model is implemented in an algorithm that allows the stress analysis of any 2D no-tension body to be directly carried out without the need of any incremental procedure (Sec. 3.1). Upon discretization of the problem, the constrained minimization of the objective function is carried out through mathematical programming [24]. If required, the entire load-displacement curve under monotonic loading can be derived at any degree of accuracy by running a sequence of independent analyses. A few comments on the proposed algorithm and comparisons with alternative optimization tools available in the literature are made in Sec. 3.2. The possibility of estimating the collapse load of the structure using an expressly developed algorithm is shown in Sec. 3.3. The effectiveness of the model in predicting the collapse load of various no-tension structures is assessed in Sec. 4. A preliminary discussion is made on the accuracy of the results obtained checking the no-tension condition only at the centroid of any finite element, or at each of the Gauss points of the element. Comparisons between the numerically estimated collapse loads and the values obtained using limit analysis specialized to no-tension materials are also presented. Finally, in Sec. 5 the main findings of the work are summarized and future extensions of the research are outlined. 


\section{Problem formulation}

According to Bruggi [9], the equilibrium of any 2D isotropic linear elastic no-tension solid can be stated in weak form as follows:

$$
\begin{cases}\min _{\rho_{1}, \rho_{2}} & \frac{1}{2} \int_{\Omega} \mathbf{D}\left(\rho_{1}, \rho_{2}, \theta\right) \underline{\varepsilon}(\underline{u}) \underline{\varepsilon}(\underline{u}) d \Omega \\ \text { s.t. } & \int_{\Omega} \mathbf{D}\left(\rho_{1}, \rho_{2}, \theta\right) \underline{\varepsilon}(\underline{u}) \underline{\varepsilon}(\underline{v}) d \Omega=\int_{\Gamma_{t}} \underline{t}_{0} \cdot \underline{v} d \Gamma \text { and }\left.\underline{u}\right|_{\Gamma_{u}}=\underline{u}_{0} \quad \forall \underline{v}, \\ & \theta \mid \widetilde{z}_{1}=z_{I} \text { and } \widetilde{z}_{2}=z_{I I}, \\ & \rho_{1}, \rho_{2} \mid \sigma_{I} \leq 0 \text { and } \sigma_{I I} \leq 0 \\ & 0<\rho_{\min } \leq \rho_{1}, \rho_{2} \leq 1 .\end{cases}
$$

In Eq. (1), $\Omega$ is the domain occupied by the solid, $\Gamma_{t}$ is its free boundary and $\Gamma_{u}$ its fixed boundary. $\underline{t}_{0}$ are prescribed tractions on $\Gamma_{t}$ and $\underline{u}_{0}$ are prescribed displacements on $\Gamma_{u}$; body forces are neglected. $\underline{u}$ is the displacement field and $\underline{\varepsilon}=\left[\varepsilon_{11} \varepsilon_{22} 2 \varepsilon_{12}\right]$ is the array of the strain components in a global Cartesian reference system $O z_{1} z_{2}$. The real isotropic no-tension material is replaced by an equivalent orthotropic material, with symmetry axes $\widetilde{z}_{1}$ and $\widetilde{z}_{2}$, coinciding with the principal stress directions, $z_{I}$ and $z_{I I}$, at any point of the real solid. Indeed, from the optimal design of anisotropic elastic solids (see e.g. [19, 21, 22]), it is well known that the symmetry axes characterizing a maximum (or a minimum) stiffness layout are aligned to the principal stress directions. Unlike the approach followed in [9], where this alignment was iteratively enforced within the solution procedure, here the orientation field $\theta\left(z_{1}, z_{2}\right)$ is added to the unknown variables, and the expected alignment of symmetry axes and principal stress directions spontaneously arises from the minimization procedure.

The stress-strain law for the equivalent material is written as $\underline{\sigma}=\mathbf{D} \underline{\varepsilon}$, where $\underline{\sigma}=\left[\sigma_{11} \sigma_{22} \sigma_{12}\right]$ and $\mathbf{D}$ can be expressed as

$$
\mathbf{D}=\mathbf{T}(\theta) \widetilde{\mathbf{D}} \mathbf{T}(\theta)^{T}
$$


being $\widetilde{\mathbf{D}}$ the elasticity matrix in the reference system $O \widetilde{z}_{1} \widetilde{z}_{2}$ and $\mathbf{T}$ a transformation matrix, depending on the orientation $\theta\left(z_{1}, z_{2}\right)$. Assuming plane stress conditions, $\widetilde{\mathbf{D}}$ reads

$$
\widetilde{\mathbf{D}}=\frac{1}{1-\widetilde{\nu}_{12} \widetilde{\nu}_{21}}\left[\begin{array}{ccc}
\widetilde{E}_{1} & \widetilde{\nu}_{12} \widetilde{E}_{2} & 0 \\
\widetilde{\nu}_{21} \widetilde{E}_{1} & \widetilde{E}_{2} & 0 \\
0 & 0 & \widetilde{G}_{12}\left(1-\widetilde{\nu}_{12} \widetilde{\nu}_{21}\right)
\end{array}\right]
$$

where $\widetilde{E}_{1}, \widetilde{E}_{2}$ are the Young's moduli of the equivalent material along $\widetilde{z}_{1}$ and $\widetilde{z}_{2}$, respectively, $\widetilde{G}_{12}$ is the in-plane shear modulus and $\widetilde{\nu}_{12}, \widetilde{\nu}_{21}$ are Poisson's ratios. The equality $\widetilde{\nu}_{12} \widetilde{E}_{2}=\widetilde{\nu}_{21} \widetilde{E}_{1}$ holds. $\mathbf{T}$ can be written as

$$
\mathbf{T}=\left[\begin{array}{ccc}
\cos ^{2} \theta & \sin ^{2} \theta & -2 \cos \theta \sin \theta \\
\sin ^{2} \theta & \cos ^{2} \theta & 2 \cos \theta \sin \theta \\
\cos \theta \sin \theta & -\cos \theta \sin \theta & \cos ^{2} \theta-\sin ^{2} \theta
\end{array}\right]
$$

The nondimensional "material densities" $\rho_{1}$ and $\rho_{2}(\in(0,1])$ define the decrease of the elastic properties of the equivalent material with respect to those of the real material along $\widetilde{z}_{1}$ and $\widetilde{z}_{2}$ if one or both principal stresses are tensile, according to a generalization of the so-called SIMP material model (see e.g. $[6,7])$ :

$$
\widetilde{E}_{1}=\rho_{1}^{p} E, \quad \widetilde{E}_{2}=\rho_{2}^{p} E, \quad \widetilde{G}_{12}=\rho_{1}^{p} \rho_{2}^{p} \frac{E}{2(1+\nu)}, \quad \widetilde{\nu}_{12}=\rho_{2}^{p} \nu, \quad \widetilde{\nu}_{21}=\rho_{1}^{p} \nu
$$

In Eq. (5), $E$ and $\nu$ are the Young's modulus and the Poisson's ratio of the real isotropic material, respectively, and $p$ is a penalization parameter (usually taken equal to 3 ). The normalized densities are given a strictly positive lower bound, $\rho_{\min }$, to avoid any singularity in the stiffness matrix of the body, $\mathbf{K}$, when a finite element discretization is adopted. 


\section{Finite element analysis of no-tension structures}

\subsection{Direct analysis for any prescribed compatible load}

The discretized form of the minimization problem (1) is implemented into a finite element program through a procedure called Solve, which allows the analysis of a linear elastic no-tension structure to be directly carried out for any prescribed load compatible with the mechanical behavior of the material.

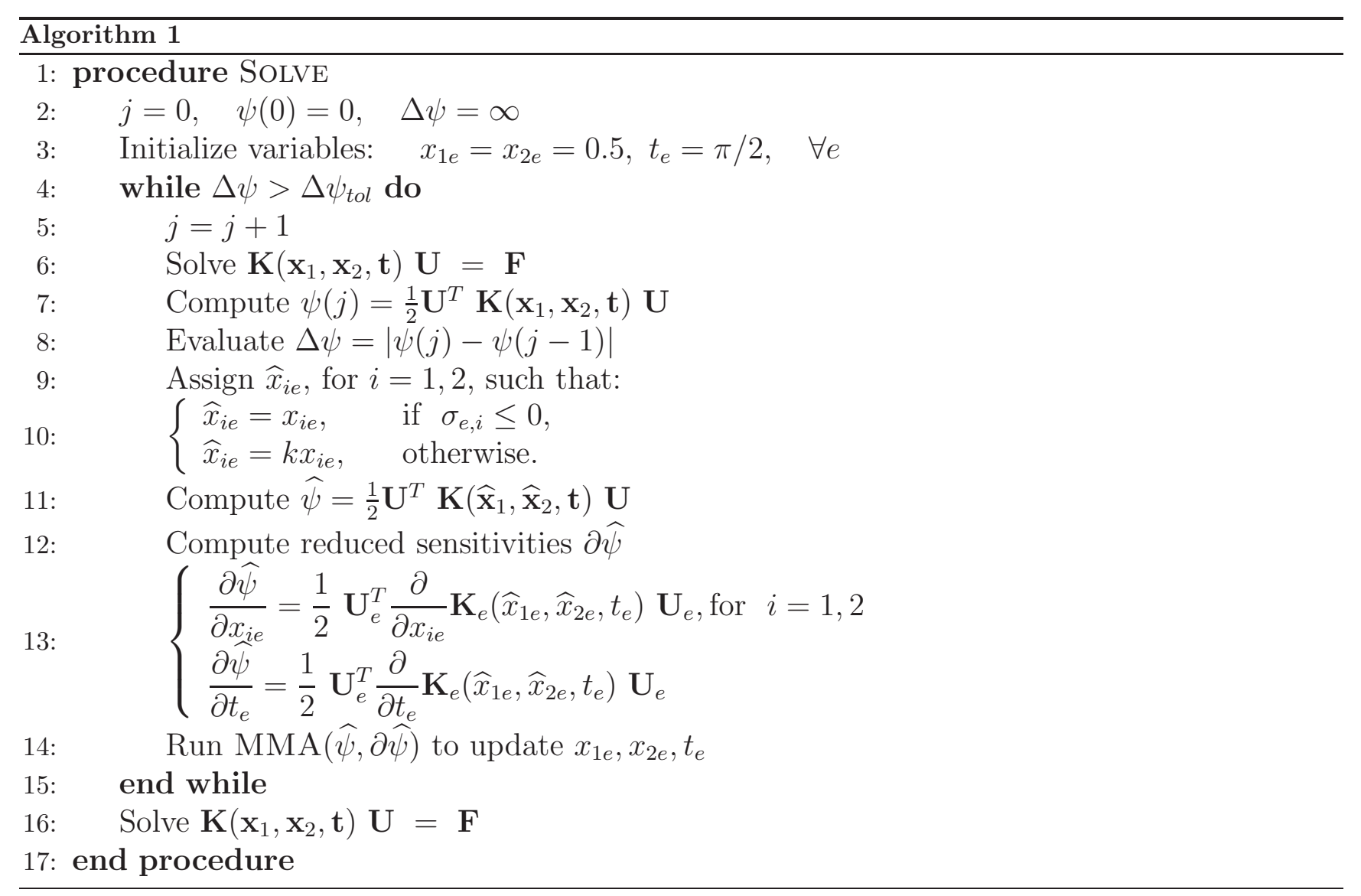

The numerical method is detailed in Algorithm 1. The minimization unknowns $x_{1 e}$ and $x_{2 e}$ are the material densities along the symmetry axes of the equivalent orthotropic material in any finite element $e$, whereas $t_{e}$ is the unknown orientation of these axes with respect to the global reference 
system. In the numerical applications shown in Sec. 4, which make use of 4-node quadrilateral Serendipity finite elements, two different discretizations are used for the three sets of variables $x_{1 e}$, $x_{2 e}$ and $t_{e}$ : an element-wise constant discretization, in which the variables are basically computed at the centroid of each element, and a bi-linear scheme, in which the values of the variables are computed at the four Gauss points of each element. For sake of illustration, the notation of Algorithm 1 refers to the former case, since $x_{1 e}, x_{2 e}$ and $t_{e}$ are scalar variables. The extension of the notation to the latter case is straightforwardly obtained introducing three arrays of four unknowns each for the $e$-th finite element.

$\mathbf{K}_{e}$ denotes the stiffness matrix of any element, $\mathbf{K}$ is the assembled stiffness matrix of the structure, symbolically expressed as $\mathbf{K}=\sum_{e=1}^{N} \mathbf{K}_{e}\left(x_{1 e}, x_{2 e}, t_{e}\right), \mathbf{x}_{\mathbf{1}}, \mathbf{x}_{\mathbf{2}}$ and $\mathbf{t}$ are three arrays collecting the unknown values of the minimization variables in all the elements, $\mathbf{U}$ is the array of the unknown degrees of freedom, $\mathbf{F}$ is the array of the equivalent nodal forces, and $k$ is a nondimensional parameter defined hereafter.

At iteration $j=0$, the minimization variables $\mathbf{x}_{1}, \mathbf{x}_{2}$ and $\mathbf{t}$ are given arbitrary starting values, assigned by the user. The array storing the values of the strain energy $\psi$ throughout the steps of the minimization procedure is initialized, as well as the scalar quantity $\Delta \psi$ that records the change in energy between two subsequent iterations (lines 2-3).

A main set of instructions is repeated within the loop of lines 4 to 15 to iteratively provide the gradient-based minimizer, herein the Method of Moving Asymptotes MMA [24], with suitable information on the objective function $\psi$ and its sensitivities, until $\Delta \psi$ is less than a prescribed tolerance $\Delta \psi_{t o l}$. At each iteration $j$, the stiffness matrix $\mathbf{K}\left(\mathbf{x}_{1}, \mathbf{x}_{2}, \mathbf{t}\right)$, depending on the current set of variables, is assembled and inverted to compute the current array of the unknown degrees of freedom $\mathbf{U}$ and the relevant value of the elastic compliance $\psi(j)$, see lines 6-7. The penalized densities $\widehat{x}_{1 e}$ and $\widehat{x}_{2 e}$ are introduced (lines 9-10) for a straightforward computation of a modified strain energy $\widehat{\psi}$, in which the terms related to any possible positive principal stress $\sigma_{e, i}, i=I, I I$, 
are reduced by a parameter $k<1$, herein $k=0.5$. Providing the MMA with the reduced objective function $\widehat{\psi}$ and the relevant sensitivities, $\partial \widehat{\psi} / \partial x_{1 e}, \partial \widehat{\psi} / \partial x_{2 e}$ and $\partial \widehat{\psi} / \partial t_{e}$, the gradientbased minimizer updates the variables $\mathbf{x}_{1}, \mathbf{x}_{2}$ and $\mathbf{t}$ and prevents any distribution of stiff material along the weak direction(s) of the no-tension material without enforcing the more demanding constraints of Eq. (1.4), see [1]. It must be remarked that the computation of the derivatives of $\widehat{\psi}$ with respect to the minimization variables is straightforward. Indeed, as usually done in topology optimization for minimum compliance problems, the sensitivity information can be computed through the adjoint method [7] without the need for any additional inversion of the current stiffness matrix, see line 13.

In case the stopping criterion $\Delta \psi \leq \Delta \psi_{t o l}$ is met, the stiffness matrix $\mathbf{K}$ is assembled with the optimal values of the minimization variables, and the displacement field solving the no-tension elasticity problem is computed (line 16).

To work with the modified strain energy $\widehat{\psi}$, a switch from the original set $x_{1 e}, x_{2 e}$ to the penalized densities $\widehat{x}_{1 e}$ and $\widehat{x}_{2 e}$ is required, depending on the sign of the principal stresses $\sigma_{e, I}$ and $\sigma_{e, I I}$ at one or more points within the element. As the material symmetry axes of the orthotropic material are aligned to the principal stress directions, denoting by $\underline{\widetilde{\sigma}}=\left[\sigma_{I} \sigma_{I I} 0\right]$ the array of the stress components evaluated in the material reference system, one has:

$$
\underline{\widetilde{\sigma}}_{e}=\widetilde{\mathbf{D}}\left(x_{1 e}, x_{2 e}\right) \mathbf{T}\left(t_{e}\right)^{T} \underline{\varepsilon}_{e},
$$

where $\mathbf{T}\left(t_{e}\right)$ and $\widetilde{\mathbf{D}}\left(x_{1 e}, x_{2 e}\right)$ can be derived from Eq.s (3-4), substituting the variable fields $\rho_{1}$, $\rho_{2}, \theta$ with discrete unknowns defined either at the centroid or at the integration points of each element.

It must be finally pointed out that most of the available numerical methods dealing with the non-linear analysis of no-tension solids (e.g., masonry) make use of incremental and iterative approaches. For any prescribed value of the applied load, Algorithm 1 solves the equilibrium 
through a one-shot minimization, i.e. an iterative non-incremental procedure. This procedure is especially suited for the analysis of no-tension structures in earthquake-prone areas at the Serviceability Limit State (SLS) or at the Damage Control Limit State (DCLS). For this class of structures, the assessment of both equilibrium and admissibility of the displacement field with respect to serviceability or damage constraints are requested for a prescribed value of the seismic excitation.

\subsection{Remarks}

The numerical method implemented in Algorithm 1 solves a minimization problem according to a formulation of topology optimization for minimum compliance. As many numerical procedures that have been successfully adopted in the literature to cope with this class of problems, the proposed approach is a gradient-based one, resorting to sequential convex programming and the analytical computation of the sensitivities. At each iteration, through a dual method the MMA solves a sequence of simpler approximate sub-problems, which are separable, convex and constructed according to the sensitivity information at the current step, as well as to the iteration history. This approach has been found to be effective in solving large scale problems, giving results in agreement with those achieved through optimality criteria, see e.g. [8]. Readers are referred to

[9] for numerical investigations assessing the convergence of a similar optimization algorithm to the analytical solution of benchmark no-tension problems.

As the objective function is non-convex, local minima might be found rather than the expected global minimum. To overcome this numerical issue, a multi-start strategy can be adopted by simply running procedure SoLve with different starting guesses. This was done in the numerical applications shown in Sec. 4, for which the same results were obtained starting the procedure with different values of the minimization variables $\left(x_{1 e}=x_{2 e}=\rho_{\min }, t_{e}=\pi\right.$ or $x_{1 e}=x_{2 e}=1, t_{e}=0$, in addition to the original assignment at line 3). Alternatively, local minima can be avoided by adopting the global convergent version of the gradient-based minimizer (GCMMA) [25]: this 
version is based on conservative convex separable approximations of the objective function, for which it is proved that the sequence of iteration points converges toward the set of Karush-KuhnTucker points. As shown in Sec. 4.1, convergence of procedure Solve needs for a limited number of iterations, meaning that either strategy can be implemented to handle numerical simulations at a reasonable computational cost.

The proposed approach is especially conceived to deal with bodies made of linear elastic notension material undergoing small displacements and strains. In principle, a similar numerical strategy could be applied to analyze structural elements made of no-compression materials, tensegrity structures consisting of separate members in pure compression and pure tension, or no-tension structures undergoing large displacements. In these applications, the "topology optimization problem" should be reformulated to accommodate geometrical nonlinearities, adding remarkable complexity to the algorithm: the linear system that has to be solved when evaluating the objective function (see line 6) should be replaced by a nonlinear system of equations, whereas the stiffness matrix and the sensitivities of the problem (see line 13) would become harder to compute. Reference is also made to the gradient-based algorithm recently presented in [16] to deal with the topology optimization of geometrically nonlinear structures.

Gradient-based approaches can be adopted in topology optimization if analytical sensitivities of the design variables are straightforwardly available, as in the case considered herein (see line 13). Alternative gradient estimations are usually considered to be computationally too expensive, because of to the typically high dimensionality of the problems addressed (see e.g. [8]). Many efforts are being directed towards the development of heuristic methods specifically conceived to deal with objective functions whose analytical sensitivities are not available or difficult to obtain (see in particular [3]). Readers are also referred to [12] for an updated survey including the adoption of heuristic methods to solve problems of structural and multidisciplinary topology optimization. 


\subsection{Approximation of the collapse load}

History plots for increasing values of the load multiplier(s) can be straightforwardly achieved running independent analyses performed through the procedure Solve at different load steps. Representing the behavior of a no-tension structure through a diagram relating the displacement of a control point to the value of the multiplier of the live loads, one gets a monotonic curve ending with a horizontal plateau. This corresponds to the activation of a mechanism, meaning that the Collapse Limit State (CLS) has been reached.

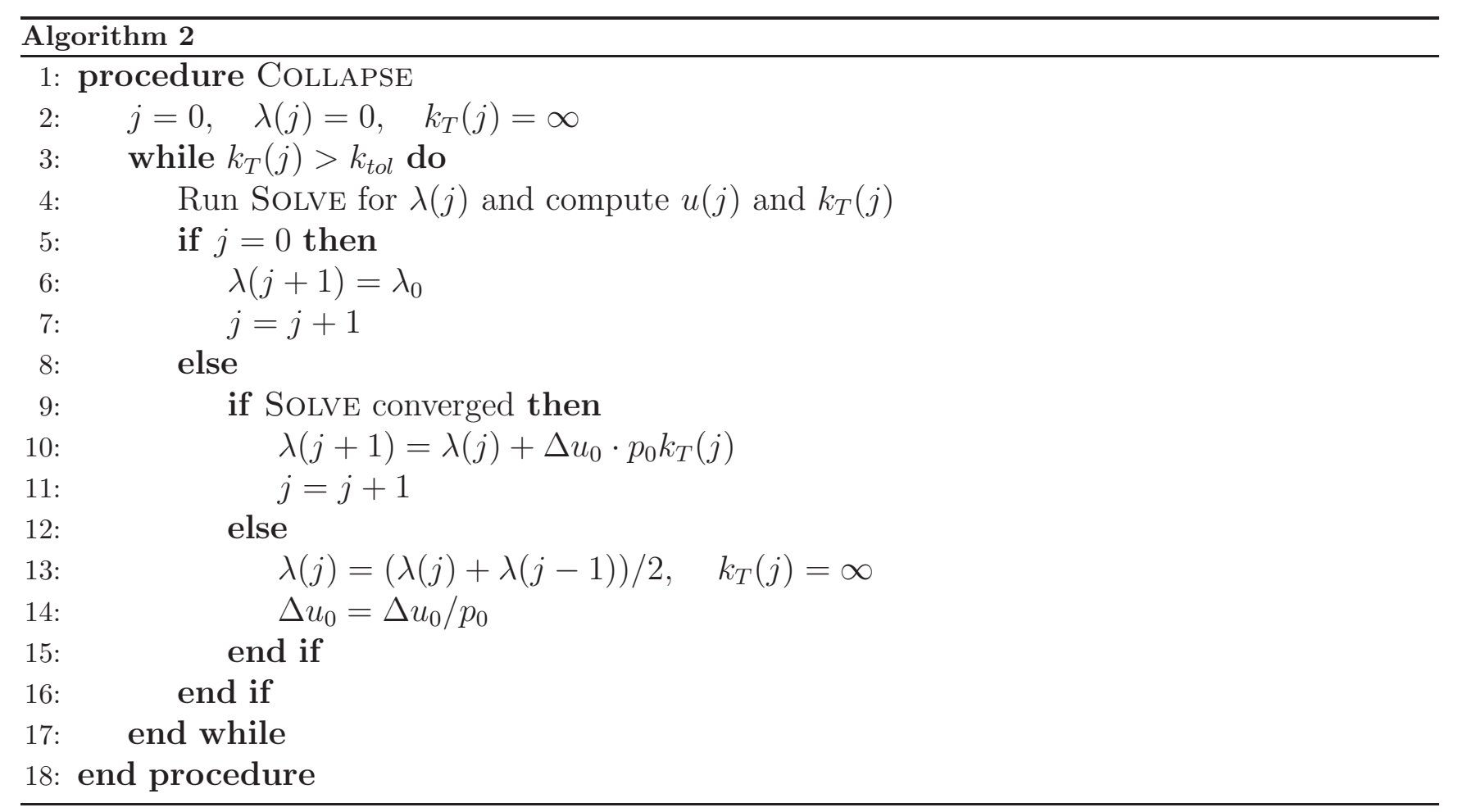

In some instances, the entire incremental response of a no-tension structure under increasing loads might not be required, as only the collapse load and the relevant failure mechanism are of interest. European building codes do not provide any specific method to capture the failure mechanisms of masonry buildings (see e.g. [13]). The Italian code adopts the approach originally 
presented in [14], which assumes deformation to be small at incipient collapse and implements limit load analysis for no-tension materials.

The expected monotonic decrease in tangent stiffness and the non-incremental feature of the algorithm SOLve are both exploited in a procedure called CollaPse, which estimates the collapse load through the set of instructions detailed in Algorithm 2.

The iterative procedure is driven by the current slope of the monotonic load-displacement curve, $k_{T}(j)$. The set of instructions at lines 4 to 16 is repeated until the current tangent stiffness $k_{T}(j)$ falls below a prescribed threshold $k_{t o l} \approx 0$, thus detecting the incipient collapse. At each load step, this parameter can be straightforwardly computed through one inversion of the current stiffness matrix and the subsequent evaluation of the displacement of the control point for any $\lambda$ through procedure SOLVE.

To start the analysis, the behavior of the structure under the dead loads only $(j=0)$ is investigated. The loop at lines 4 to 16 is entered after initializing $k_{T}(j)$ at line 2 and computing the solution for $\lambda=0$, including the relevant displacement of the control point $u(j)$ and the current tangent stiffness $k_{T}(j)$, at line 4 .

The subsequent iteration $(j=1)$ is accomplished for a user-defined initial load multiplier equal to $\lambda_{0}$, see lines 6-7. Once again, procedure Solve is used to solve equilibrium and compute the current values of $u(j)$ and $k_{T}(j)$ according to line 4 .

At each step $j \geq 1$, convergence of the procedure Solve is checked to assess the equilibrium of the structure under the loads corresponding to the current multiplier $\lambda(j)$, see line 9 . If $\operatorname{SoLvE}$ converges, the structure can bear an increased load, meaning that a further step can be defined along with the relevant load multiplier $\lambda$ (lines 10-11). The increment of the load multiplier is computed as $\Delta u_{0} \cdot p_{0} k_{T}(j)$, where $\Delta u_{0}$ is a user-defined displacement increment, $p_{0}<1$ accounts for the expected decrease in overall stiffness, and $k_{T}(j)$ works similarly to the generalized stiffness parameter introduced in [26]. Conversely, if Solve does not converge, the bearing capacity of the 
no-tension structure has been exceeded and the $j$-th step must be repeated. A bi-section method between the last converged load multiplier $\lambda(j-1)$ and the current one is adopted to update $\lambda(j)$, whereas the assignment $k_{T}(j)=\infty$ prevents the algorithm to stop if convergence is not achieved (line 13). Also, the user-defined displacement increment $\Delta u_{0}$ is increased with the only aim of speeding up convergence to the collapse load (line 14).

Depending on the outcome of the check at line 9, the subsequent call of procedure SolvE computes the structural response for an increased value of the load multiplier ( $\operatorname{step} j+1$ ) or for a decreased one (repetition of step $j$ due to loss of convergence in the previous run). The instructions at lines 4 to 16 are iteratively repeated accounting for the expected monotonic decrease of the load-displacement curve through the evolving tangent stiffness $k_{T}(j)$ and the possible update of the user-defined parameter $\Delta u_{0}$.

It must be emphasized once more that, unlike conventional methods, in principle the above procedure does not require the definition of the entire load-displacement curve to predict the collapse load. Indeed, only few points of the curve can be computed by the algorithm CoLLAPSE to capture incipient failure.

Finally, note that the proposed procedure predicts the collapse mechanisms of no-tension structures assuming strains and displacements to be small. In Sec. 4.2 and 4.3 the results achieved through the linear elastic no-tension model will be shown to be in full agreement with those derived through conventional limit analysis applied to no-tension materials [17]. In its present form, the proposed method cannot be used to predict the collapse load and mechanism(s) of structures undergoing large displacements. A discussion was made in Sec. 3.2 about the possible extension of the proposed numerical framework to the case of geometrically nonlinear structures. 

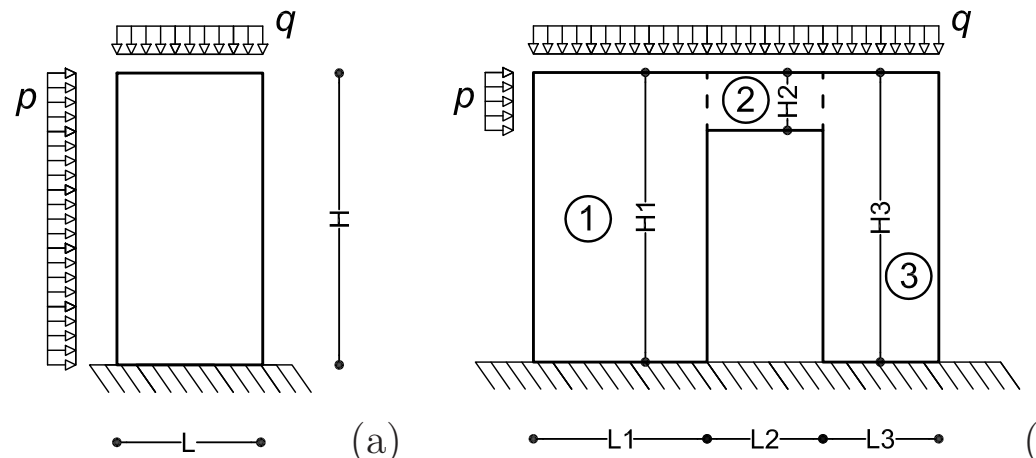

(a)

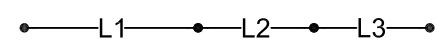

(b)

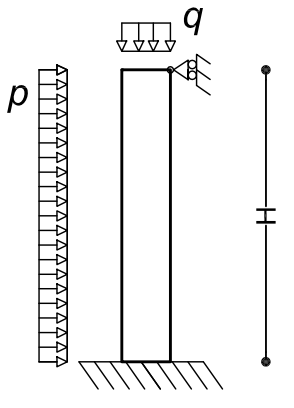

$\bullet-L \rightarrow$

(c)

Figure 1: Geometry and boundary conditions: Example 1(a), Example 2(b) and Example 3(c).

\section{Numerical applications}

\subsection{Example 1. Rectangular panel}

Consider a rectangular panel, of width $L=0.5 \mathrm{~m}$, height $H=1 \mathrm{~m}$ and thickness $t h=0.1 \mathrm{~m}$, see Figure 1(a). The mechanical parameters used in the simulations are $E=1,000 \mathrm{MPa}$ and $\nu=0.2$. An evenly distributed vertical dead load $q=20 \mathrm{kN} / \mathrm{m}$ is applied along the upper side of the panel, whereas an evenly distributed horizontal live load acts on the left side of the wall: its intensity $p$ increases with a multiplier $\lambda$ and is expressed as $p=\lambda q L / H$.

A set of numerical simulations is preliminary performed with $\lambda=0.25$ and $\lambda=0.35$ to investigate the convergence features of the adopted energy-based approach considering different discretizations of the unknown fields. A control point is chosen at the midspan of the upper side of the wall: its horizontal displacement is estimated for increasing values of the mesh size parameter $h$, which is the length of the side of each of the square finite elements the adopted discretization consists of. The coarsest mesh is made of 32 four-node finite elements, whereas 512 elements are used in the finest one. Two kinds of discretizations are adopted for the "normalized material density" fields, $\rho_{1}$ and $\rho_{2}$, and for the orientation $\theta$, as discussed in Sec. 3. Figure 2 shows convergence curves for a centroid-based and for a Gauss point-based discretization at $\lambda=0.25$ 

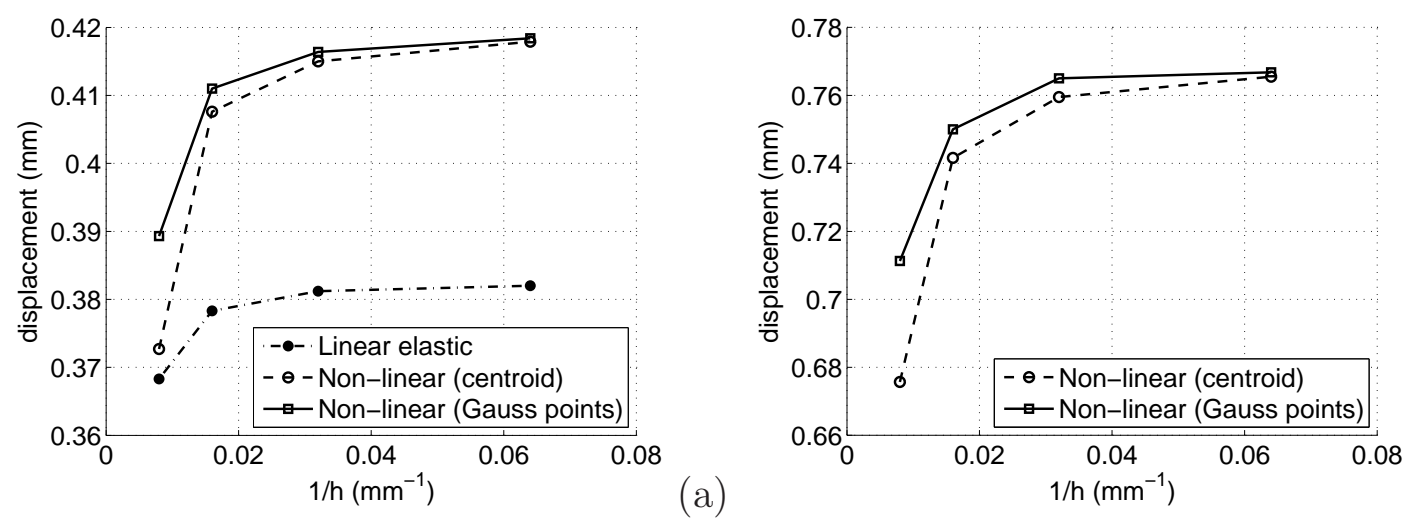

(b)

Figure 2: Example 1. Convergence of the horizontal displacement of the control point for different assumptions on the discretization of the material behavior: $\lambda=0.25$ (a) and $\lambda=0.35$ (b).

(Figure 2a) and $\lambda=0.35$ (Figure 2b).

The diagrams in Figure 2 show that, irrespective of the value of the load multiplier $\lambda$, the two discretizations predict the same asymptote as the element size decreases. For a given mesh density, the Gauss point-based approximation is more accurate than the centroid-based one, but the difference in terms of predicted displacements decreases as the mesh size parameter $h$ increases. Additionally, Figure 2(a) reports the convergence curve computed at $\lambda=0.25$ assuming the material to be linear elastic and isotropic, with the aim of providing a comparison with conventional linear analyses adopting the same bi-linear discretization of the displacement field: the increase in flexibility due to the no-tension assumption is apparent.

Referring to the case $\lambda=0.35$, Figures $3(\mathrm{a})$ and $3(\mathrm{~b})$ show the distribution of the principal stresses assuming the material to be linear elastic or no-tension, respectively. Red (respectively, blue) lines stand for tensile (resp., compressive) principal stresses. Only a reduced part of the clamped edge bears the external loads because of the no-tension constraint. The reduction in resistant section is accompanied by an increased deformability at the base of the wall (see the deformation modes in Figure 4). 

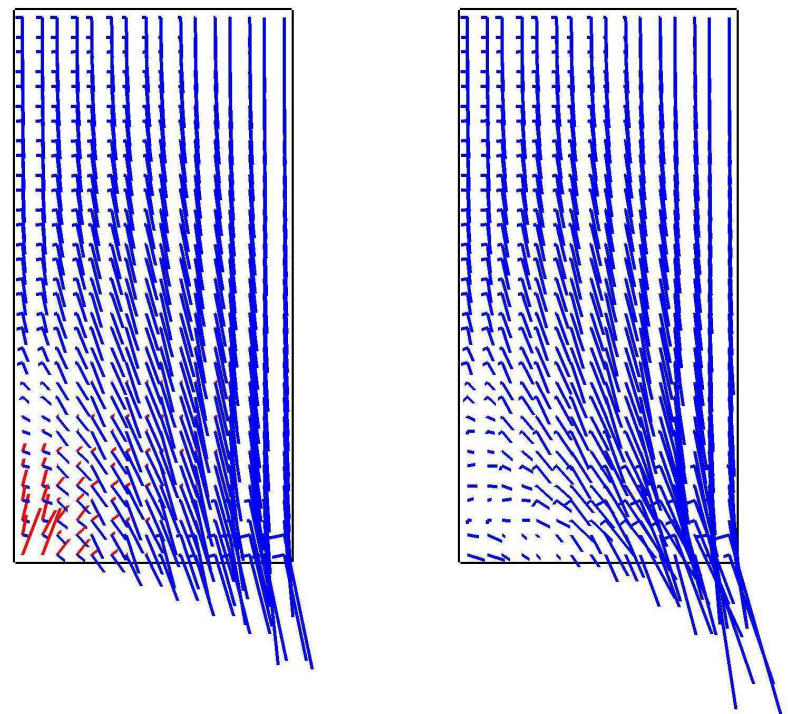

(a)

(b)

Figure 3: Example 1. Principal stress directions at the Gauss points in case of linear (a) and nonlinear (b) modeling of the material $(\lambda=0.35)$.

Finally, Figure 5 shows history plots of the strain energy, that is, the objective function for procedure SolvE. The same number of iteration is approximately needed to achieve equilibrium both with the Gauss point-based and with the centroid-based discretizations. It must be remarked, however, that the required CPU time is not the same, because of the different number of minimization unknowns involved in the two schemes, and is definitely higher in the former case.

\subsection{Example 2. In-plane loaded walls}

The carrying capacity of the windowed panel shown in Figure 1(b) is sought. The values of the geometrical parameters $L_{1}, L_{2} \ldots H_{3}$ defining the structural element are listed in Table 1 . The thickness of brickwork is $0.35 \mathrm{~m}$. The panel is supposed to consist of an isotropic no-tension material, with a Young's modulus $E=2,500 \mathrm{MPa}$ and a Poisson's ratio $\nu=0.2$ : these values are appropriate, for instance, for solid brick masonry if anisotropy is neglected. As shown in Figure 

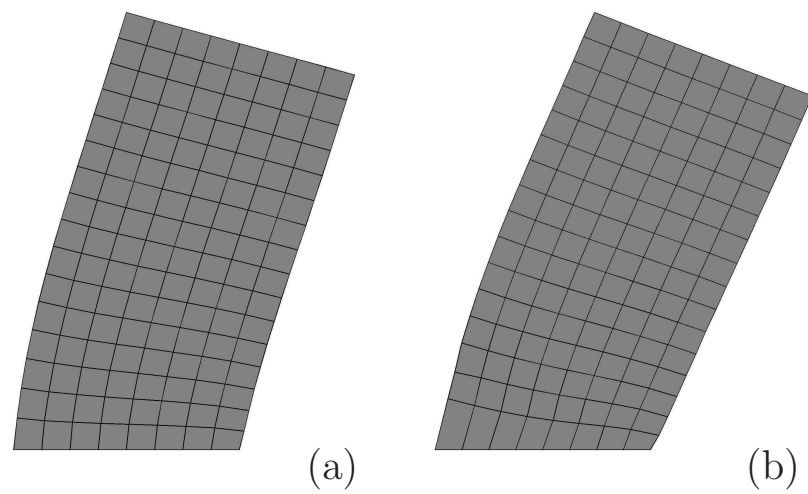

(b)

Figure 4: Example 1. (Magnified) deformed shape in case of linear (a) and nonlinear (b) material modeling $(\lambda=0.35)$.

\begin{tabular}{cc}
\hline $\mathrm{L} / \mathrm{H}$ & \\
\hline$L_{1}$ & $1.80 \mathrm{~m}$ \\
$L_{2}$ & $1.20 \mathrm{~m}$ \\
$L_{3}$ & $1.20 \mathrm{~m}$ \\
$H_{1}$ & $3.00 \mathrm{~m}$ \\
$H_{2}$ & $0.60 \mathrm{~m}$ \\
$H_{3}$ & $3.00 \mathrm{~m}$ \\
\hline
\end{tabular}

Table 1: Example 2. Geometry of the windowed panel.

1(b), the upper side of the panel is acted upon by a vertical uniform pressure $q=15 \mathrm{kN} / \mathrm{m}$, representing the dead load due to the overtopping storeys. A horizontal live load $p$ is applied along the height of the lintel, and is expressed as:

$$
p=\lambda q\left(L_{1}+L_{2}+L_{3}\right) / H_{2} \text {. }
$$

The top-right corner of the windowed panel is taken as control point, and its horizontal displacement is monitored to characterize the global behavior of the structure as $\lambda$ increases.

A fixed-step algorithm is first adopted to assess the accuracy of procedure SolvE in predicting 


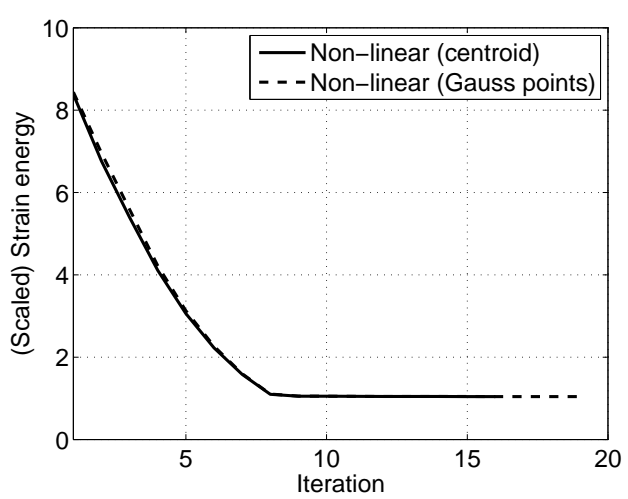

Figure 5: Example 1. History plot of the strain energy for different interpolations of the properties of the equivalent orthotropic material; $\lambda=0.35$.

the collapse load of the structure. The minimization variables are computed at the centroid of each element. Performing subsequent independent analyses with procedure Solve, assuming $\Delta \lambda=0.01$ 35 steps are positively accomplished. The value of the load multiplier and the displacement of the control point are both recorded at the end of each converged simulation. If Solve does not convergence (herein for $\lambda>0.36$ ), the relevant results are ignored and no additional load increment is applied.

Figure 6 shows the load-displacement curve computed through the fixed-step algorithm, along with the set of points found implementing the algorithm CoLlapse with the following setup: $\lambda_{0}=0.1, \Delta u_{0}=3 \mathrm{~mm}, p_{0}=0.5$ and $k_{t o l}=0.005 \mathrm{~mm}^{-1}$. After computing the solution under the dead load $(j=0)$ and at the initial fixed increment $\lambda_{0}(j=1)$, according to line 10 of Algorithm 2 the load multiplier would attain the value $\lambda_{(j=2)}=0.506$ in the subsequent step, at which no convergence is found. Hence, the step is repeated and the load multiplier is updated to $\lambda_{(j=2)}=0.303$, following the bi-section criterion of line 13 . Computing the progressive decrease in the current tangent stiffness at each iteration, only five additional steps based on the updated value of $\Delta u_{0}$ (see line 14) are needed to meet the stopping criterion: the approximate collapse load 


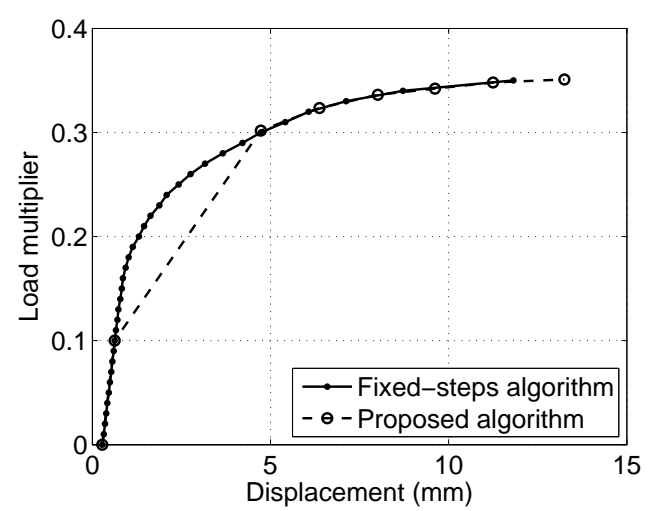

Figure 6: Example 2. Horizontal displacement of the top-right corner of the panel versus load multiplier $\lambda$ : fixedstep algorithm vs. proposed algorithm.

multiplier is $\lambda_{c}=\lambda_{(j=7)}=0.353$.

The effect of a different choice for the user-defined parameters is investigated in Figure 7, comparing the load--displacement curve shown in Figure 6 with the results obtained by halving $\lambda_{0}$ or $\Delta u_{0}$. In the former case, the same number of iterations is needed to predict the collapse load with respect to the reference setup $\left(\lambda_{0}=0.1, \Delta u_{0}=3 \mathrm{~mm}\right)$. In the latter, less than the 20 steps allow the plateau of the curve to be captured, versus the 35 increments required by the fixed-step algorithm.

Figure 8(a) shows the distribution of the principal (compressive) stresses arising under the vertical dead load only, that is at $\lambda_{j=0}=0$. Note the typical arch-like stress distribution in the architrave, which originates non-trivial stress fluxes within the adjacent walls. At $\lambda_{j=1}=0.10$ the base of the lateral walls is still fully compressed (Figure $8(\mathrm{~b})$ ), whereas, at $\lambda_{j=3}=0.303$ their resistant section is significantly reduced (see Figure 8(c)), with a dramatic decrease in the overall stiffness.

The areas where both principal stresses are tensile basically behave as "void regions" [9], where any positive semi-definite "cracking strain" can develop. Figure 8(c) shows that only small regions 


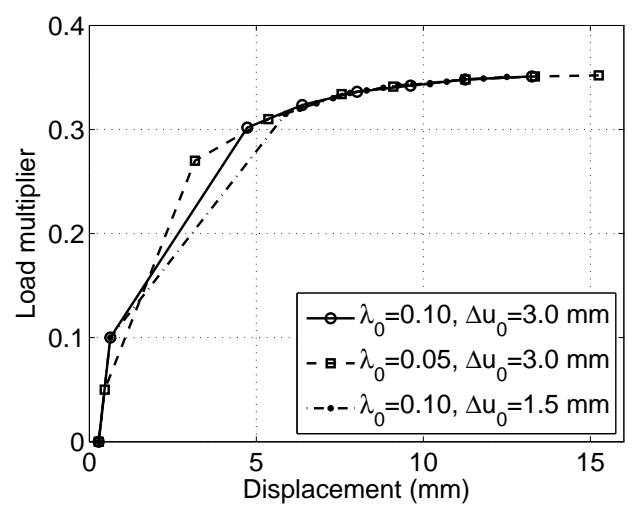

Figure 7: Example 2. Horizontal displacement of the top-right corner of the panel versus load multiplier $\lambda$ : effect of the values of the user-defined parameters $\lambda_{0}$ and $\Delta u_{0}$ on the numerical load-displacement curve.

between the lintel and the lateral walls are stressed: this basically means that the lintel can rotate with respect to the walls, around the two corners where inclined reactions are transmitted between these elements. As the load multiplier increases, the extension of the stress-released regions within the walls increases, with an additional drop in the overall stiffness.

Eventually, the solution shown in Figure $8(\mathrm{~d})$ is attained at $\lambda_{j=7}=0.354$. Two more hinges are formed at the lower right corners of the walls, thus allowing for the activation of a collapse mechanism. The relevant (magnified) deformed geometry is shown in Figure 9(a). Keeping in mind the stress plot of Figure $8(\mathrm{~d})$, it is straightforward to approximate this deformation mode with the rigid block mechanism sketched in Figure 9(b). Neglecting the elastic strains found in the regions experiencing compressive stresses, the two walls (blocks 1 and 3) and the lintel (block 2) can be seen as three rigid bodies connected by four hinges: the windowed panel degenerates into a partially constrained structure, i.e. a collapse mechanism. According to the literature, this is a classical collapse mechanism for bearing walls connected by lintels [14].

The analytical collapse load multiplier $\lambda_{a}$ of the rigid block mechanism in Figure 9(b) is computed in Appendix A.1 by limit analysis, and is equal to 0.3545. This is in good agreement with 

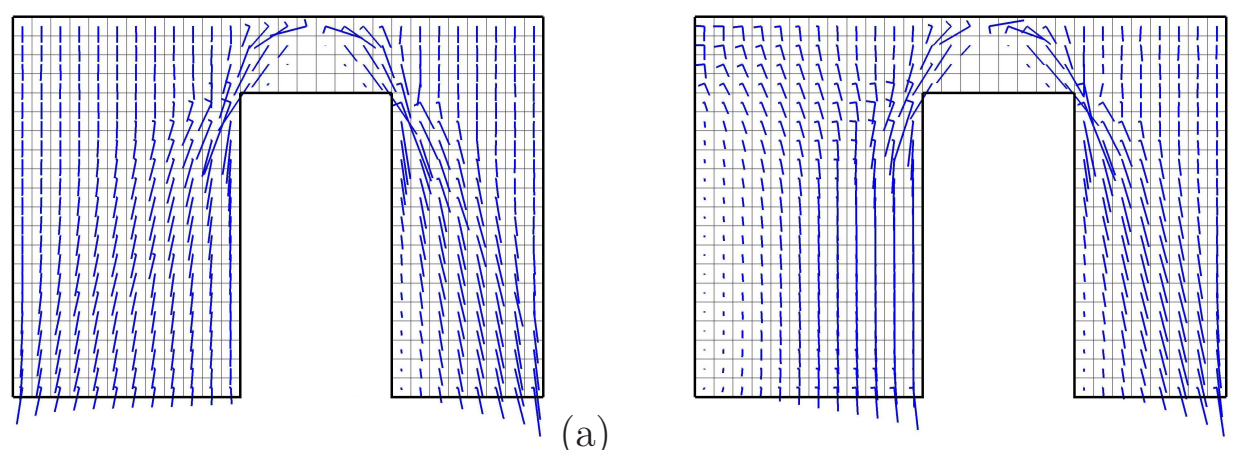

(a)

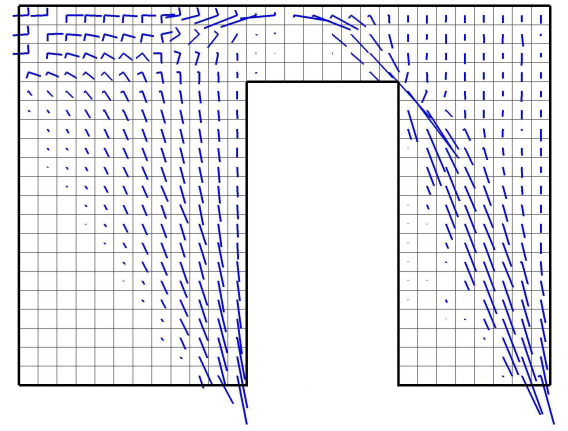

(c)

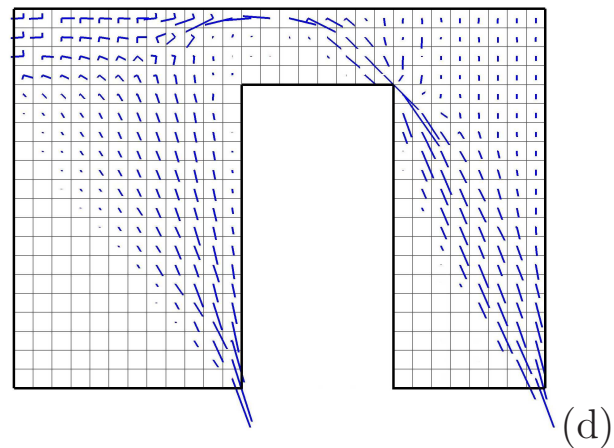

Figure 8: Example 2. Principal (compressive) stress directions at different values of the load multiplier: $\lambda=0$ (a), $\lambda=0.10(\mathrm{~b}), \lambda=0.303(\mathrm{c}), \lambda_{c}=0.365(\mathrm{~d})$.

the approximated value given by the proposed numerical procedure, $\lambda_{c}=\lambda_{(j=7)}=0.353$.

The resistance of a masonry structure against horizontal actions can be increased by inserting steel tie rods within the lintel and connecting the two piers at the same height. The tie has the aim of preventing the failure mechanism presented in Figure 9(b). The strengthening effect of a tie rod can be qualitatively modeled by applying a uniform pre-stress over the regions where the end-plates would be located. To this purpose, an additional investigation is carried out on the panel of Figure 1(b) including a compressive stress of $0.2 \mathrm{MPa}$ applied at the top of both vertical edges of the panel, over areas equal to $H_{2} \cdot t h$.

Figure 10(a) shows the distribution of the principal stresses in the reinforced panel under the 

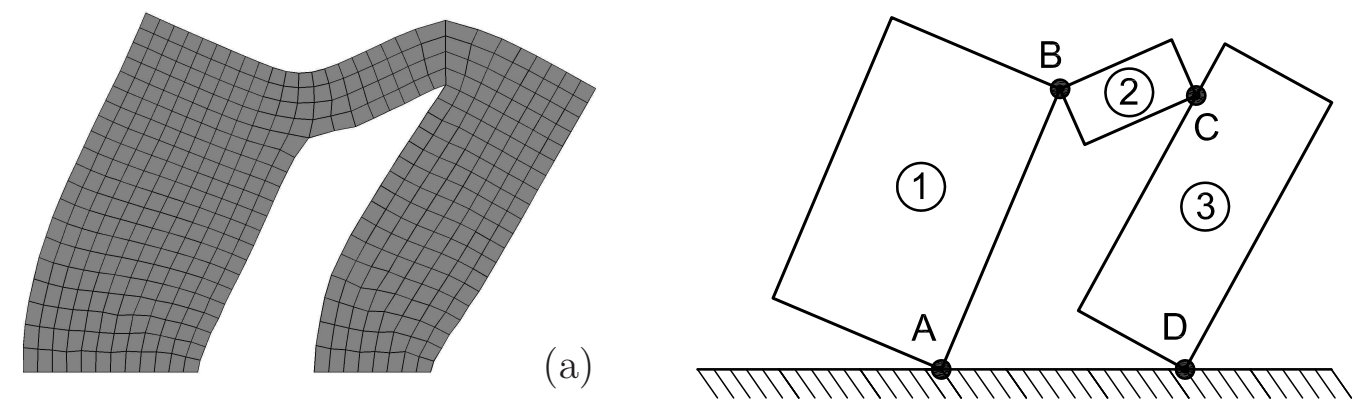

(b)

Figure 9: Example 2. (Magnified) computed deformed geometry at $\lambda_{c}=0.353$ (a) vs. analytically computed collapse mechanism (collapse load multiplier $\lambda_{a}=0.3545$ (b)).

effect of the vertical dead load only. Whereas the lintel in the unreinforced architrave behaves like an arch (Figure 8(a)), the applied pre-stress provides the lintel with a beam-like behavior, and no significant horizontal reaction is transmitted to the ground. Figure 10(b) shows the compressive stress fluxes at the last step of a numerical investigation implementing the updated boundary conditions through the algorithm CollAPSE, at $\lambda_{c}=0.554$. The applied pre-stress makes the stress in the lintel and in the upper parts of the walls nearly uniform. Two main struts cross the lateral walls diagonally, whereas the remaining parts are virtually unstressed. A hinge can activate at the foot of each strut, as can be inferred by the shape of the (magnified) deformed structure shown in Figure 11(a). Neglecting once again the elastic strain in the compressed regions, a partially restrained structure consisting of mutually hinged rigid blocks can be identified (see Figure 11(b)). Appendix A.2 provides the collapse load multiplier computed for this mechanism by limit analysis $\left(\lambda_{a}=0.561\right)$, in good agreement with that predicted by algorithm CoLLAPSE $\left(\lambda_{c}=0.554\right)$. The increase in terms of collapse load (around $\left.50 \%\right)$ with respect to the unreinforced solution is remarkable.

\subsection{Example 3. Out-of-plane loaded wall}

A rectangular panel of width $L=0.5 \mathrm{~m}$, height $H=3 \mathrm{~m}$ and thickness $t h=1 \mathrm{~m}$ is considered, see Figure 1(c). It consists of a no-tension material with the same mechanical parameters used in 


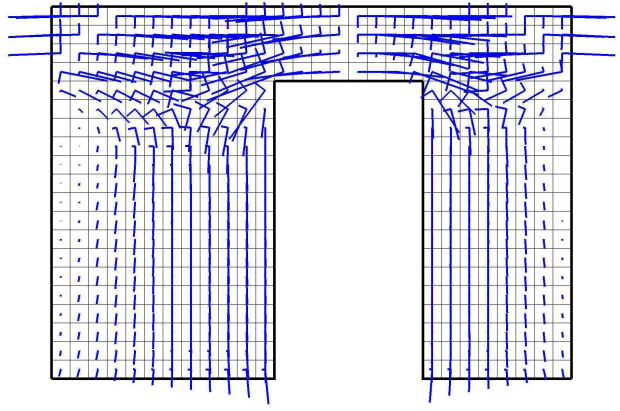

(a)

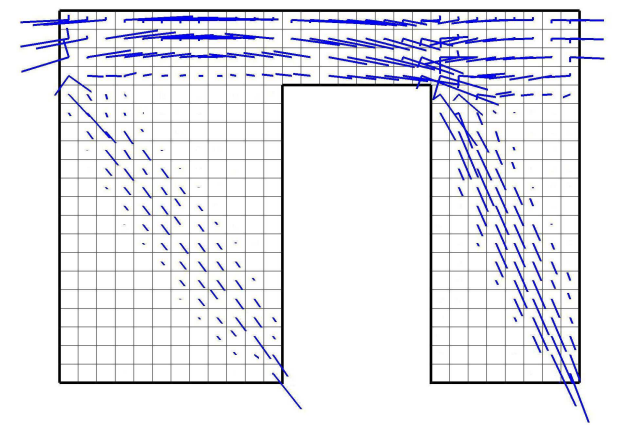

(b)

Figure 10: Example 2 with enforced pre-stress. Principal (compressive) stress directions at different values of the load multiplier: $\lambda=0$ (a), $\lambda_{c}=0.10(\mathrm{~b})$.
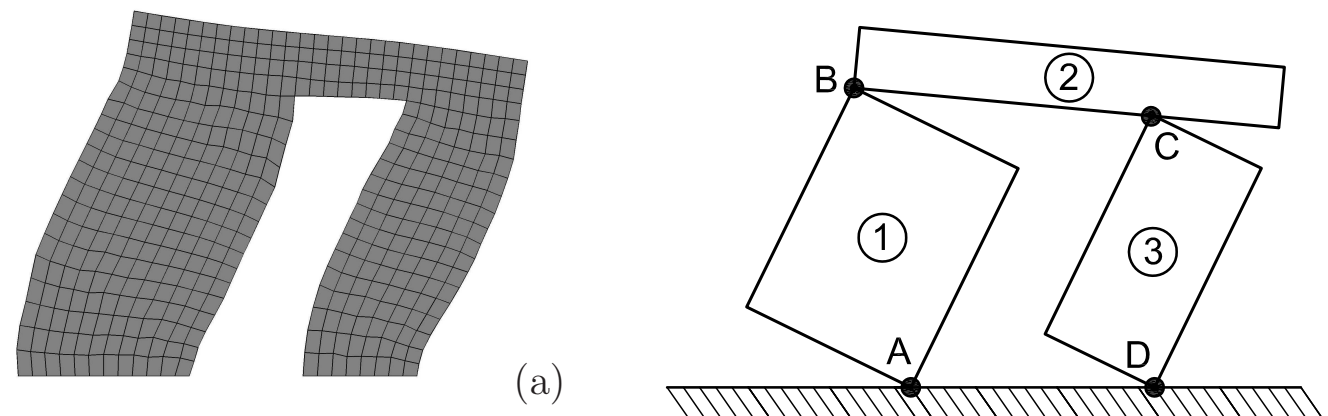

(b)

Figure 11: Example 2 with enforced pre-stress. (Magnified) computed deformed geometry for $\lambda_{c}=0.554$ (a) vs. theoretical collapse mechanism at the analytically computed collapse load multiplier $\lambda_{a}=0.561$ (b).

the previous example. A uniform vertical dead load $q=60 \mathrm{kN} / \mathrm{m}$ is applied along the upper side of the panel; the left side of the panel is loaded by a uniform pressure $p=\lambda q / 6$, where $\lambda$ is a load multiplier. This configuration aims at simulating the out-of-plane behavior of a unit section of any masonry wall experiencing seismic actions, as prescribed by technical codes. The base of the panel is clamped to the ground, whereas storeys are expected to provide an effective horizontal restraint along the whole upper side.

Figure 12 shows the approximated load-displacement curve computed through the algorithm Collapse with $\lambda_{0}=0.1, \Delta u_{0}=3 \mathrm{~mm}, p_{0}=0.5$ and $k_{t o l}=0.005 \mathrm{~mm}^{-1}$, assuming the minimiza- 


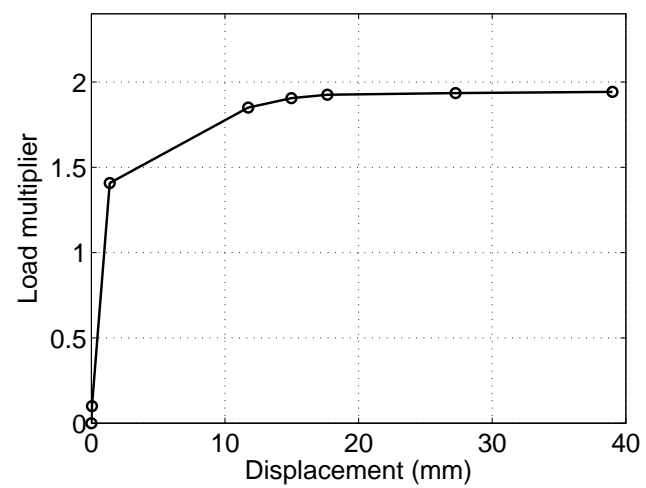

Figure 12: Example 3. Horizontal displacement at half of the the height of the panel versus load multiplier $\lambda$.

tion variables to be constant within each element. Similarly to the previous examples, the solutions under the dead load $(j=0)$ and at the first load increment $\lambda_{0}(j=1)$ are initially evaluated. The tangent stiffness computed for $j=1$ leads to the subsequent load step at $\lambda_{(j=2)}=2.714$, at which no convergence is found. Hence, the step is repeated with a load multiplier reduced to $\lambda_{(j=2)}=1.407$, according the bi-section criterion of line 13 in Algorithm 2. Computing at each step the progressive decrease in tangent stiffness with the updated increment $\Delta u_{0}=6 \mathrm{~mm}$, three additional steps are processed before convergence is lost again at $\lambda_{(j=5)}=1.955$. Updating the load multiplier and doubling $\Delta u_{0}$ once more, the algorithm meets the stopping criterion and approximates the collapse load at $\lambda_{c}=\lambda_{(j=7)}=1.935$.

Figure 13(a) shows the distribution of the principal (compressive) stresses at $\lambda_{j=0}=0.10$. The panel is fully stressed, and a non-uniform reaction is found over the base. Figure 13(b) shows the stress state at step $j=2$, at which most of the lower part of the panel is "cracked", whereas approximately half of the midspan section is compressed. Figure 13(c) shows the principal stresses computed at the estimated collapse multiplier. The flux of compressive stresses found in the lower and in the central region of the panel is confined within a very limited part of the relevant sections, meaning that two hinges are expected to activate for any additional infinitesimal increase in the 


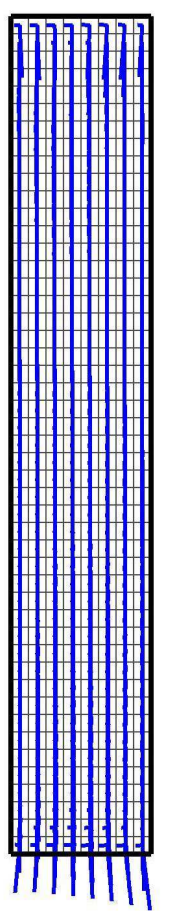

(a)

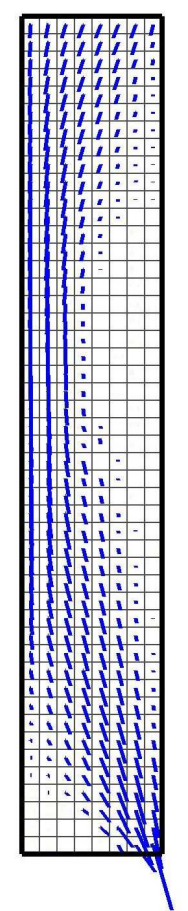

(b)

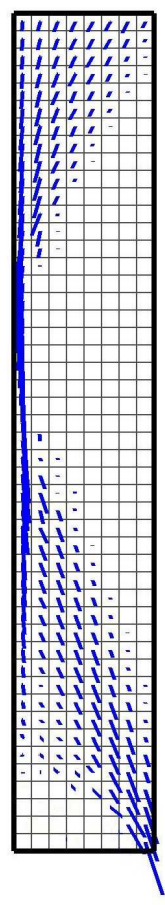

(c)

Figure 13: Example 3. Principal (compressive) stress directions at different values of the load multiplier: $\lambda=0.10$ (a), $\lambda=1.407$ (b), $\lambda=1.935$ (c).

live load (see the comments in Sec. 4.2).

The (magnified) deformed geometry of the panel at the estimated collapse load is shown in Figure 14(a). Again, neglecting elastic strains, the panel can be replaced by a mechanism consisting of two rigid blocks connected by an internal hinge (Figure 14(b)). The lower block is hinged to the ground at $A$, whereas the upper block is supported at the top. The collapse load of this mechanism is computed in Appendix A.3 by the upper bound theorem of limit analysis: the location of the internal hinge $B$ is varied along the height of the wall to minimize the kinematic multiplier. This gives an analytical collapse multiplier $\lambda_{a}=1.943$, which is well matched by that 


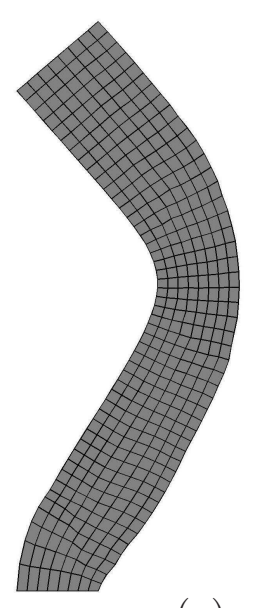

(a)

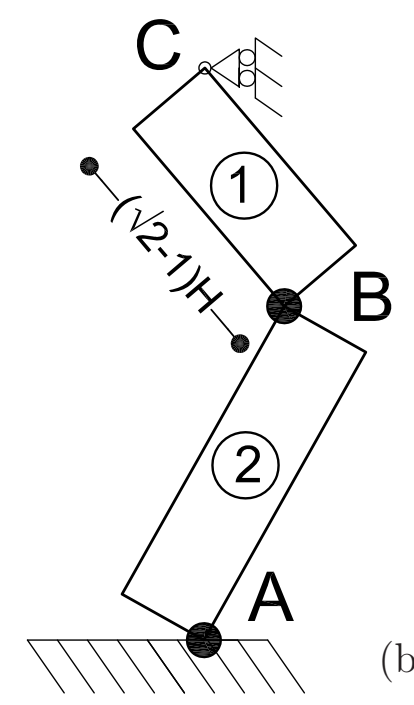

(b)

Figure 14: Example 3. (Magnified) computed deformed geometry at $\lambda_{c}=0.935$ (a) vs. theoretical collapse mechanism at the analytically computed collapse load multiplier $\lambda_{a}=1.943$ (b).

computed numerically $\left(\lambda_{c}=\lambda_{(j=7)}=1.935\right)$. Figure $14(\mathrm{~b})$ shows the position of hinge $B$ according to the analytical computations, in good agreement with the findings of the numerical simulation shown in Figure 14(a).

\section{Conclusions}

Following recent proposals from the literature addressing energy-based methods for the direct analysis of linear elastic no-tension solids, a numerical method is presented to perform the analysis of isotropic 2D masonry-like structures acted upon by monotonic loads and undergoing small displacements and strains.

A key-point of energy-based methods is that they are able to solve the elastic equilibrium of no-tension solids for any given load disregarding the previous load history. This has been exploited in the formulation of a numerical scheme to predict the collapse behavior of no-tension masonry-like structures without recovering the full load-displacement curve. In agreement with 
the assumptions of classical limit analysis [17] and the provisions of building codes, the deformation of the considered masonry structures is assumed to be small even at incipient collapse.

The proposed algorithm correctly captures the in- and out-of-plane collapse mechanisms predicted by limit analysis applied to no-tension bodies, without any a-priori hypothesis regarding the type of collapse mechanism or the position of the "plastic hinges" (Sec. 4.3). It can also take the presence of reinforcing ties into account (Sec. 4.2).

Owing to the non-incremental nature of the one-shot energy-based procedure implemented in Solve (Sec. 3), different choices for the parameters can be made in running the algorithm Collapse (sec. 3.3) without any loss of robustness. For instance, the value of $\lambda_{0}$ could be defined so as to embed within the numerical procedure the assessment of the structure at the Serviceability Limit State (SLS) or at the Damage Control Limit State (DCLS), whereas $\Delta u_{0}$ could be roughly related to the accuracy requested in the definition of the plateau of the load-displacement curve. Also, if the whole curve has to be numerically determined, parameter $p_{0}$ can be further reduced to add points in the initial part of the diagram.

As shown in Sec. 4.1, if a coarse mesh is adopted, the most accurate solution is obtained computing the minimization variables at the four Gauss points of each finite element. As the size of the elements decreases, a simpler approach in which the minimization unknowns are computed only at the centroid of each element gives equally accurate results. The former version, which is computationally more expensive, is preferable for analyses in which the evaluation of the displacements and of the stresses in the structure under prescribed loads is required, in particular at the SLS or at the DCLS. The latter version, which is computationally cheaper but equally reliable in capturing the reduction in resistant section of the structural members, can be efficiently used to detect the collapse mechanism and the collapse load multiplier of the structure.

In the continuation of the work, the extension of the proposed approach to the 3D case will be dealt with, with the aim of analyzing massive and vaulted masonry structures. The simplification 
adopted so far, according to which masonry is macroscopically isotropic, will also be removed to take elastic anisotropy into account.

\section{Appendix A. Limit analysis computations}

The specialization of Limit Analysis to no-tension structures has been extensively discussed e.g. by Heyman [17]. According to this author, $(i)$ deformations are assumed to be small at incipient collapse, $(\mathrm{ii})$ the material is assumed to be rigid-perfectly plastic, (iii) its compressive strength is supposed to be unlimited, $(i v)$ its tensile strength is neglected and $(v)$ "tensile strains" are supposed to be unbounded.

Limit Analysis is adopted herein to evaluate the bearing capacity of some of the no-tension structures considered in Sec. 4. The collapse load is evaluated by a kinematic approach considering 1-degree-of-freedom mechanisms consisting of rotating rigid blocks. According to Heyman's assumptions, the internal virtual power vanishes and equilibrium of the partially constrained structure reads $\dot{W}_{\text {ext }}=0$.

\section{Appendix A.1. Example 2}

The collapse load multiplier $\lambda_{a}$ for the unreinforced windowed panel of Example 2 is herein computed enforcing the equilibrium of the partially constrained structure made by three rigid blocks, represented in Figure A.15, through the Principle of Virtual Power. The potentially critical sections are assumed to be at the base of the panel and at the interfaces between lintel and lateral walls.

Denote by $O_{i}$ the instantaneous center of rotation (i.c.r.) of the $i$-th block $(i=1,2,3$ - see Figure A.15) and by $\omega_{i}$ its instantaneous velocity. Blocks 1 and 3 undergo clockwise instantaneous rotations, whereas block 2 rotates anticlockwise. Setting $\overline{O_{1} B} \omega_{1}=\overline{O_{2} B} \omega_{2}$, one gets

$$
\omega_{2}=\frac{H_{1} L_{3}}{\left(L_{2}+L_{3}\right)\left(H_{3}-H_{2}\right)-H_{1} L_{3}} \omega_{1}
$$




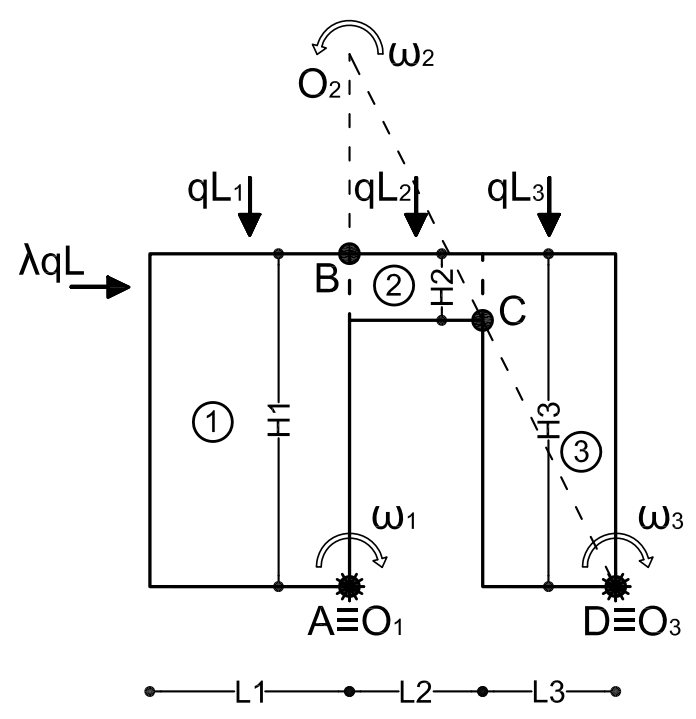

Figure A.15: Example 2. Unreinforced windowed panel: kinematics for the analytical computation of the collapse load multiplier.

Similarly, setting $\overline{\mathrm{O}_{2} C} \omega_{2}=\overline{\mathrm{O}_{3} C} \omega_{3}$ and exploiting Eq. (A.1), one gets:

$$
\omega_{3}=\frac{L_{2}}{L_{3}} \omega_{2}=\frac{H_{1} L_{2}}{\left(L_{2}+L_{3}\right)\left(H_{3}-H_{2}\right)-H_{1} L_{3}} \omega_{1}
$$

The power of the external forces reads:

$$
\dot{W}_{e x t}=\lambda q L \omega_{1}\left(H_{1}-\frac{H_{2}}{2}\right)-q L_{1} \omega_{1} \frac{L_{1}}{2}-q L_{2} \omega_{2} \frac{L_{2}}{2}-q L_{3} \omega_{3} \frac{L_{3}}{2}
$$

where $L=L_{1}+L_{2}+L_{3}$. Setting $\dot{W}_{\text {ext }}=0 \forall \omega_{1}$, replacing Eq.s (A.1-A.2) in Eq. (A.3) and simplifying for $\omega_{1}$, the analytical expression of the collapse load multiplier is obtained:

$$
\lambda_{a}=\frac{1}{L\left(2 H_{1}-H_{2}\right)}\left(L_{1}^{2}-\frac{H_{1} L_{2} L_{3}^{2}+H_{1} L_{2}^{2} L_{3}}{\left(L_{2}+L_{3}\right)\left(H_{2}-H_{3}\right)+H_{1} L_{3}}\right)
$$

which gives $\lambda_{a}=0.354$ with the values of the geometrical parameters listed in Table 1 . 
Appendix A.2. Example 2 (with pre-stress)

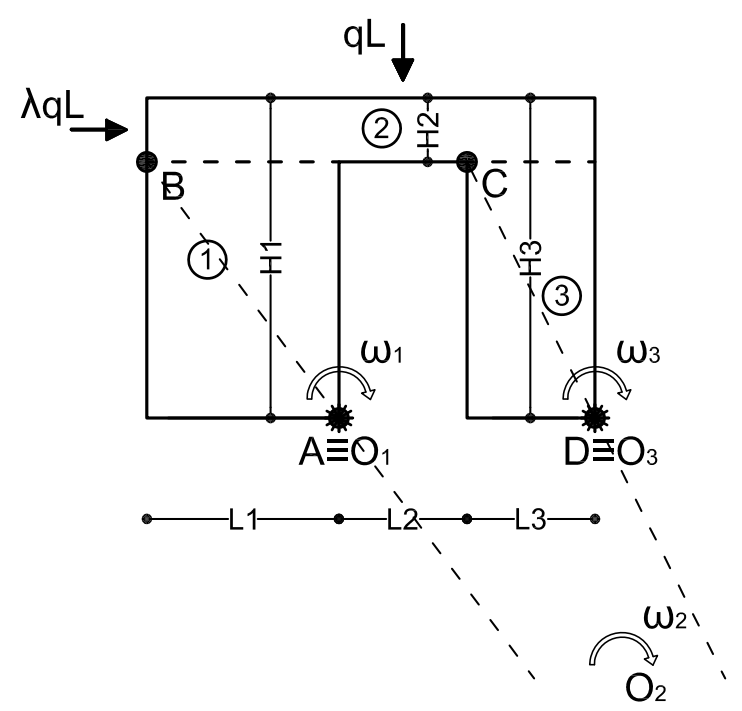

Figure A.16: Example 2. Pre-stressed windowed panel: kinematics for the computation of the collapse load multiplier.

Similar calculations are now carried out to evaluate the collapse load multiplier $\lambda_{a}$ for Example 2, when a pre-stress is applied to the architrave. The collapse mechanism of the structure is supposed to consist of three rigid blocks, as shown in Figure A.16.

The i.c.r.s $O_{1}, O_{2}$ and $O_{3}$ of the three blocks are shown in Figure A.16. The coordinates of $O_{2}$ in a Cartesian reference system with origin at $B$ are:

$$
x_{O 2}=\frac{L_{1}\left(L_{1}+L_{2}\right)\left(H_{2}-H_{3}\right)}{\left(H_{1}-H_{2}\right) L_{3}+\left(H_{2}-H_{3}\right) L_{1}} \quad \text { and } \quad y_{O 2}=\frac{\left(L_{1}+L_{2}\right)\left(H_{2}-H_{3}\right)\left(H_{1}-H_{2}\right)}{\left(H_{1}-H_{2}\right) L_{3}+\left(H_{2}-H_{3}\right) L_{1}} .
$$

Blocks 1, 2 and 3 undergo clockwise instantaneous rotations with angular velocity $\omega_{1}, \omega_{2}$ and $\omega_{3}$, respectively. The power of the external forces can be easily computed in terms of $\omega_{2}$. No 
contribution is due to the self-balanced pre-stress forces. Equilibrium can be enforced as:

$$
\dot{W}_{e x t}=\lambda q L \omega_{2}\left(y_{O 2}+\frac{H_{2}}{2}\right)-q L \omega_{2}\left(x_{O 2}-\frac{L}{2}\right)=0 \forall \omega_{2}
$$

where $L=L_{1}+L_{2}+L_{3}$. Substituting Eq. (A.5) in Eq. (A.6) and simplifying for $\omega_{2}$, the collapse load multiplier is found:

$$
\lambda_{a}=\frac{2 L_{1}\left(H_{2}-H_{3}\right)\left(L_{1}+L_{2}\right)-L_{1}\left(H_{2}-H_{3}\right) L-L_{3}\left(H_{1}-H_{2}\right) L}{H_{2} L_{1}\left(H_{2}-H_{3}\right)+H_{2} L_{3}\left(H_{1}-H_{2}\right)+2\left(H_{1}-H_{2}\right)\left(H_{2}-H_{3}\right)\left(L_{1}+L_{2}\right)} .
$$

This gives $\lambda_{a}=0.561$ with the values of the geometrical parameters listed in Table 1 .

Appendix A.3. Example 3

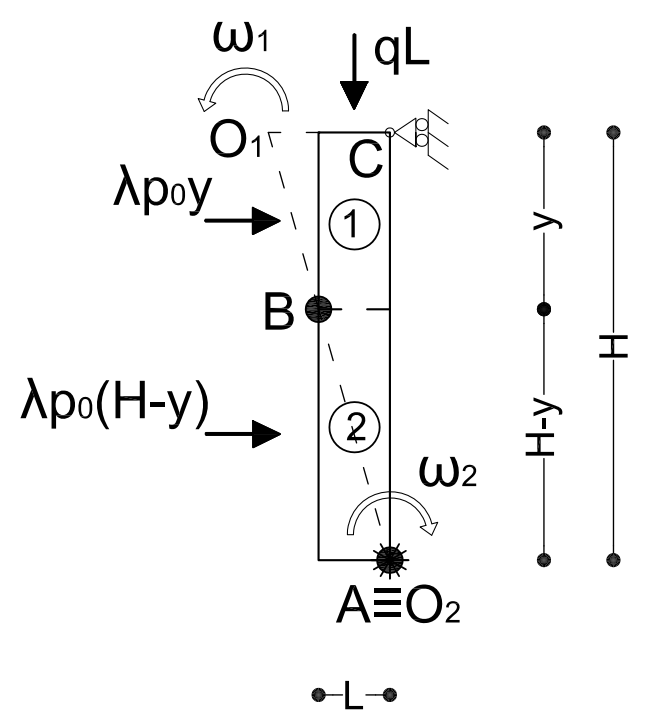

Figure A.17: Example 3. Kinematics for limit load analysis.

The collapse load multiplier $\lambda_{a}$ for the transversely loaded panel of Example 3 is herein computed enforcing the equilibrium of the partially constrained structure made by two rigid blocks 
represented in Figure A.17. Unlike the previous examples, the position $(y)$ of the critical section separating the two blocks, in which the internal hinge $B$ activates, is not known a-priori.

The i.c.r.s $O_{1}$ and $O_{2}$ of the two blocks are shown in Figure A.17. Block 2 undergoes clockwise instantaneous rotation with velocity $\omega_{2}$, whereas block 1 rotates anticlockwise with instantaneous velocity $\omega_{1}$. Setting $\overline{O_{1} B} \omega_{1}=\overline{O_{2} B} \omega_{2}$, one gets:

$$
\omega_{2}=\frac{y}{H-y} \omega_{1}
$$

The power of the external forces reads:

$$
\dot{W}_{e x t}=\lambda p_{0} y \omega_{1} \frac{y}{2}+\lambda p_{0}(H-y) \omega_{2} \frac{H-y}{2}-q L \omega_{1}\left(\frac{L y}{H-y}+\frac{L}{2}\right) .
$$

Equilibrium of the partially constrained structure requires $\dot{W}_{\text {ext }}=0 \forall \omega_{1}$. Substituting Eq. (A.8) in Eq. (A.9) and simplifying for $\omega_{1}$, one gets:

$$
\lambda=\frac{q L^{2}}{p_{0} H} \frac{H+y}{H y-y^{2}}
$$

The ordinate $y$ of $B$ in the real collapse mechanism minimizes the kinematic multiplier and is given by $y_{a}=(\sqrt{2}-1) H$. Accordingly, the collapse multiplier is:

$$
\lambda_{a}=\frac{q L^{2}}{p_{0} H^{2}}(2 \sqrt{2}+3)
$$

With the values of the geometrical parameters detailed in Section 4.3, one gets $y_{a}=1.243 \mathrm{~m}$ and $\lambda_{a}=1.943$. 


\section{References}

[1] Ananiev, S., 2005. On equivalence between optimality criteria and projected gradient methods with application to topology optimization problem. Multibody System Dynamics 13(1), 25-38.

[2] Angelillo, M., Cardamone, L., Fortunato, F., 2010. A numerical model for masonry-like structures. Journal of the Mechanics of Materials and Structures 5(4), 583-615.

[3] Aulig, N., Menzel, S., Nutwell, E., Detwiler, D., 2014. Towards multi-objective topology optimization of structures subject to crash and static load cases. Proceedings of The 4th International Conference on Engineering Optimization EngOpt2014, Lisbon, Portugal, 8-11 September $2014,847-852$.

[4] Baratta, A., Corbi, O., 2010. An approach to masonry structural analysis by the no-tension assumption - Part I: Material modeling, theoretical setup, and closed form solutions. Applied Mechanics Reviews 63(4), 040802.

[5] Baratta, A., Corbi, O., 2010. An approach to masonry structural analysis by the no-tension assumption - Part II: Load singularities, numerical implementation and applications. Applied Mechanics Reviews 63(4), 040803.

[6] Bendsøe, M., Kikuchi, N., 1988. Generating optimal topologies in structural design using a homogenization method. Computer Methods in Applied Mechanics and Engineering 71, 197224 .

[7] Bendsøe, M., Sigmund. O., 1999. Material interpolation schemes in topology optimization. Archives of Applied Mechanics 69, 635-654.

[8] Bendsøe, M.P., Sigmund, O., 2003. Topology Optimization - Theory, Methods and Applications. Springer, Berlin. 
[9] Bruggi, M., 2014. Finite element analysis of no-tension structures as a topology optimization problem. Structural and Multidisciplinary Optimization 50(6), 957-973.

[10] Bruggi, M., Milani, G., Taliercio, A. 2013. Design of the optimal fiber-reinforcement for masonry structures via topology optimization. International Journal of Solids and Structures 50(13), 2087-2106.

[11] Cuomo, M., Ventura, G., 2000. Complementary energy formulation of no tension masonry-like solids. Computer Methods in Applied Mechanics and Engineering 189(1), 313-339.

[12] Deaton, J., Grandhi, R., 2014. A survey of structural and multidisciplinary continuum topology optimization: post 2000. Structural and Multidisciplinary Optimization 49(1), 1-38.

[13] Magenes, G., Penna, A., 2009. Existing masonry buildings: general code issues and methods of analysis and assessment. Proceedings of the Workshop Eurocode 8: perspectives from the Italian Standpoint, Naples, Italy, 3 April 2009, 185-198.

[14] D'Ayala, D., Speranza, E., 2003. Definition of Collapse Mechanisms and Seismic Vulnerability of Historic Masonry Buildings. Earthquake Spectra, 19(3), 479-509.

[15] Del Piero, G., 1989. Constitutive equation and compatibility of the external loads for linear elastic masonry-like materials. Meccanica, 24(3), 150-162.

[16] Gomes, F.A.M., Senne, T.A., 2014. An algorithm for the topology optimization of geometrically nonlinear structures. International Journal for Numerical Methods in Engineering 99(6), 391-409.

[17] Heyman, J., 1966. The stone skeleton. International Journal of Solids and Structures 2, 249279. 
[18] Lourenço, P.B., Rots, J., 1997. A multi-surface interface model for the analysis of masonry structures. ASCE Journal of Engineering Mechanics 123(7), 660-668.

[19] Pedersen N., 1989. On optimal orientation of orthotropic materials. Structural Optimization $1,101-106$.

[20] Pelà, L., Cervera, M., Roca, P., 2011. Continuum damage model for orthotropic materials: Application to masonry. Computer Methods in Applied Mechanics and Engineering 200, 917930.

[21] Rovati, M., Taliercio, A., 2003. Stationarity of the strain energy density for some classes of anisotropic solids. International Journal of Solids and Structures 40(22), 6043-6075.

[22] Seregin, G.A., Troitskii, V.A., 1981. On the best position of elastic symmetry planes in an orthotropic body. Journal of Applied Mathematics and Mechanics 45, 139-142.

[23] Shieh-Beygi, B., Pietruszczak, S., 2008. Numerical analysis of structural masonry: mesoscale approach. Computers \& Structures 86(21-22), 1958-1973.

[24] Svanberg, K., 1987. Method of moving asymptotes - A new method for structural optimization. International Journal for Numerical Methods in Engineering 24, 359-373.

[25] Svanberg, K., 2002. A Class of Globally Convergent Optimization Methods Based on Conservative Convex Separable Approximations. SIAM Journal on Optimization 12(2), 555-573.

[26] Yang, Y.-B., Shieh, M.S., 1990. Solution method for nonlinear problems with multiple critical points. AIAA Journal 28(12), 2110-2116. 